\title{
New Solutions to Nonlinear Ordinary Differential Equations
}

\author{
Moawia Alghalith \\ Economics Department, UWI, Trinidad, Cuba \\ E-mail: malghalith@gmail.com \\ Received January 11, 2011; revised January 19, 2011; accepted January 29, 2011
}

\begin{abstract}
In contrast to the Euler method and the subsequent methods, we provide solutions to nonlinear ordinary differential equations. Consequently, our method does not require convergence. We apply our method to a second-order nonlinear ordinary differential equation ODE. However, the method is applicable to higher order ODEs.
\end{abstract}

Keywords: Ordinary Differential Equations ODE; Euler’s Method

\section{Introduction}

There are several methods of solving nonlinear ordinary differential equations, such as the Euler method, RungeKutta methods and linear multistep methods. For a detailed description of these methods, see, for example, Kaw and Kalu (2009), Cellier and Kofman (2008) and Butcher (2008). However, these methods are approximations of the solution and thus they require the assumption of convergence to the solution. Consequently, numerical methods, based on real data, are needed to obtain a solution.

In this paper, in contrast to the previous methods, we present solutions to nonlinear ordinary differential equations without the requirement of convergence and without the need to numerical methods. In addition, our method is far simpler than the existing methods.

\section{The Model}

We attempt to solve the following nonlinear ordinary differential equation (higher-order equations can also be used)

$$
y^{\prime \prime}(s)=f\left(s, y^{\prime}(s), y(s)\right), s \in S,
$$

Consider the following Taylor expansion of $y$ around $\alpha$

$$
y(s)=y(\alpha)+y^{\prime}(\alpha)(s-\alpha)+\frac{1}{2} y^{\prime \prime}(\alpha)(s-\alpha)^{2}+R(s),
$$

where

$$
\begin{aligned}
R(s)= & y(s)-y(\alpha)+y^{\prime}(\alpha)(s-\alpha) \\
& +\frac{1}{2} y^{\prime \prime}(\alpha)(s-\alpha)^{2}
\end{aligned}
$$

is the remainder. Our intermediate goal is to minimize the remainder (in absolute value) with respect to time $s$

$$
\min _{s} R(s)
$$

The first-order condition yields

$$
R^{\prime}\left(s^{*}\right)=y^{\prime}\left(s^{*}\right)-y^{\prime}(\alpha)-y^{\prime \prime}(\alpha)\left(s^{*}-\alpha\right)=0,
$$

and thus

$$
y\left(s^{*}\right)=y(\alpha)+y^{\prime}(\alpha)\left(s^{*}-\alpha\right)+\frac{1}{2} y^{\prime \prime}(\alpha)\left(s^{*}-\alpha\right)^{2} .
$$

Since $y^{\prime \prime}(\alpha)=f\left(\alpha, y^{\prime}(\alpha), y(\alpha)\right)$, we obtain

$$
\begin{aligned}
y\left(s^{*}\right)= & y(\alpha)+y^{\prime}(\alpha)\left(s^{*}-\alpha\right) \\
& +\frac{1}{2} f\left(\alpha, y^{\prime}(\alpha), y(\alpha)\right)\left(s^{*}-\alpha\right)^{2} .
\end{aligned}
$$

But $y^{\prime}(\alpha)=g(\alpha, y(\alpha))$ (this is a result of integrating (1)) and thus substituting this into (1), we obtain

$$
\begin{aligned}
y\left(s^{*}\right)= & y(\alpha)+g(\alpha, y(\alpha))\left(s^{*}-\alpha\right) \\
& +\frac{1}{2} f\left(\alpha, y^{\prime}(\alpha), y(\alpha)\right)\left(s^{*}-\alpha\right)^{2} .
\end{aligned}
$$

The initial values $\alpha, y(\alpha), g(\alpha, y(\alpha))$, and $f\left(\alpha, y^{\prime}(\alpha), y(\alpha)\right)$ are known (assumed by the previous literature). Thus, this is a solution to (1). The extension of this method to higher-order differential equations is straightforward. 


\section{Practical Examples}

For simplicity, we present two first-order numerical examples. Using the above procedure, the solution for a first-order differential equation takes the form

$$
y\left(s^{*}\right)=y(\alpha)+g(\alpha, y(\alpha))\left(s^{*}-\alpha\right),
$$

and the minimization necessary condition

$$
y^{\prime}\left(s^{*}\right)-g(\alpha, y(\alpha))=0 .
$$

Example 1. $y^{\prime}(s)=s^{2}$ with initial values

$$
\alpha=1, \quad y(\alpha)=1 \text { and } g(\alpha, y(\alpha))=1 .
$$

It is well established in the literature that the solution of the differential equation depends on the initial values, and that different initial values produce different solutions. Therefore, from the necessary condition, $s^{* 2}=1$ and thus $s^{*}=-1$, since $s^{*} \neq \alpha$ by construction. Hence, using (2), we obtain

$$
y\left(s^{*}\right)=1+1 \cdot(-1-1)=-1 .
$$

Example 2. $y^{\prime}(s)=\sqrt{s}-s+1$ with initial values $\alpha=0$,

$$
y(\alpha)=0 \text { and } g(\alpha, y(\alpha))=1 .
$$

Hence, $\sqrt{s^{*}}-s^{*}+1=1$; therefore, $s^{*}=1$ and thus

$$
y\left(s^{*}\right)=0+1 \cdot(1-0)=1
$$

\section{References}

[1] J. C. Butcher, "Numerical methods for ordinary differential equations,” Wiley, W. Sussex, England, 2008.

[2] F. Cellier and E. Kofman, "Continuous system simulation,” Springer Verlag, New York, NY, 2006.

[3] A. Kaw and E. Kalu, "Numerical methods with applications,” www.autarkaw.com, 2009. 